\title{
High-field diffusion tensor imaging of mouse brain in vivo using single-shot STEAM MRI
}

\author{
Susann Boretius ${ }^{\text {a,* }}$, Jens Würfel ${ }^{b}$, Frauke Zipp ${ }^{\text {b,c }}$, Jens Frahm ${ }^{\text {a,c }}$, Thomas Michaelis ${ }^{\text {a }}$ \\ a Biomedizinische NMR Forschungs GmbH am Max-Planck-Institut für biophysikalische Chemie, 37070 Göttingen, Germany \\ ${ }^{\mathrm{b}}$ Institute of Neuroimmunology, Charité University Hospital, Berlin, Germany \\ ${ }^{\mathrm{c}}$ Institut für Multiple-Sklerose-Forschung, Bereich Humanmedizin der Universität Göttingen und \\ Gemeinnützige Hertie-Stiftung, 37073 Göttingen, Germany
}

Received 28 August 2006; received in revised form 19 October 2006; accepted 22 October 2006

\begin{abstract}
Information about the microstructural organization of cerebral white matter that is accessible by magnetic resonance diffusion tensor imaging (DTI) gains increasing importance for studies of animal brain. Particular challenges occur for in vivo conditions as well as at high magnetic fields. Here, we have employed a diffusion-weighted (DW) single-shot STEAM MRI sequence for DTI of mouse brain in vivo at 7 T. The approach exploits the increased longitudinal magnetization and prolonged $T_{1}$ relaxation times of water protons at higher magnetic field strengths without suffering from susceptibility-induced artifacts. When compared to studies at $2.35 \mathrm{~T}$, half Fourier DW STEAM MRI at $7 \mathrm{~T}$ yielded a substantial gain in signal-to-noise ratio (SNR) that could be invested either in a reduction of the measurement time or an increase of the spatial resolution. Thus, for a measurement time of $3 \mathrm{~h}$, DTI with a voxel size of $117 \mu \mathrm{m} \times 117 \mu \mathrm{m} \times 720 \mu \mathrm{m}$ not only resulted in high-quality maps of the fractional anisotropy and main diffusion direction (MDD), but also allowed for fiber tracking of major mouse brain structures in vivo.
\end{abstract}

(C) 2006 Elsevier B.V. All rights reserved.

Keywords: Magnetic resonance imaging; Diffusion tensor imaging; Fiber tractography; Mouse; Brain

\section{Introduction}

In recent years, magnetic resonance diffusion tensor imaging (DTI) has emerged as a unique tool for a noninvasive assessment of white matter microstructure. Applications deal with both human and animal brain under physiologic and pathologic conditions. The technique exploits the anisotropic nature of the water diffusion in neuroaxonal fibers. Because the water mobility is less restricted along the direction of a white matter fiber bundle and more restricted perpendicular to it where axonal membranes and myelin sheaths hinder the free diffusion, measurements of respective differences, the degree of diffusion anisotropy and the main diffusion direction (MDD) provide insights into the underlying fiber orientation and neuronal connectivity. Alterations of anisotropy and directional diffusivities have already been reported in a number of pathologies including axonal damage and demyelination, for example, see Song et

\footnotetext{
* Corresponding author. Tel.: +49 551201 1729; fax: +49 5512011307.

E-mail address: sboreti@gwdg.de (S. Boretius).
}

al. (2002); Huisman et al. (2004); Nair et al. (2005); Sun et al. (2006).

As described by Basser et al. (1994), DTI requires the acquisition of diffusion-weighted (DW) images in at least six noncolinear directions as well as one image without or with only minor diffusion encoding. From this series of images the elements of the diffusion tensor and its eigenvalues can be estimated on a voxel by voxel basis. In a technical sense, the MRI approach to diffusion relies on the attenuation of the available MRI signal in the presence of diffusion-encoding gradients. DTI is therefore especially prone to poor signal-to-noise ratio (SNR). Apart from the optimized design of MRI sequences and radiofrequency coils, the use of a high magnetic field strength remains an obvious choice for improving the SNR by an enlarged polarization of nuclear spins. Although the MRI signal - or, more precisely, the generated longitudinal magnetization - is expected to scale more than linearly with magnetic field strength (Rutt and Lee, 1996), the actual situation for in vivo proton MRI is often compromised by prolonged $T_{1}$ and markedly reduced $T_{2}$ and $T_{2}^{*}$ relaxation times at higher fields. In particular, the latter effect refers to stronger magnetic field inhomogeneities and 
therefore may enhance geometric distortions and signal losses depending on the MRI signal type acquired. Because of the physical properties of RF-refocused echoes as opposed to gradient echoes, single-shot STEAM MRI (Frahm et al., 1985; Finsterbusch and Frahm, 2002) is immune to inhomogeneity effects and - in contrast to echo-planar imaging (EPI) - may fully exploit the advantages of an increased longitudinal magnetization as well as of prolonged $T_{1}$ relaxation times without being affected by reduced $T_{2}^{*}$ (or $T_{2}$ ) values. Moreover, the technique is less prone to image distortions due to eddy currents. This is because single-shot STEAM MRI employs individually excited echoes that represent individual lines in k space, whereas EPI relies on a series of continuously reversed gradient echoes that accumulate eddy current-induced phase errors while scanning $\mathrm{k}$ space.

The purpose of this study was to demonstrate the practical potential of half Fourier diffusion-weighted single-shot STEAM MRI for DTI of mouse brain at $7 \mathrm{~T}$. Apart from mapping rotationally invariant parameters of the diffusion tensor such as the fractional anisotropy and main diffusion direction at sufficient anatomical resolution, special emphasis was paid to the feasibility of fiber tractography under in vivo conditions.

\section{Materials and methods}

\subsection{Animals}

All experiments were performed in accordance with German animal protection laws and were approved by the responsible governmental authority. A total of six adult SJL/J mice were included in the study. For MRI, anesthesia was induced with $5.0 \%$ and maintained with $1.5-2.0 \%$ isoflurane using artificial ventilation $(2.35 \mathrm{~T})$ or spontaneous breathing $(7 \mathrm{~T})$. Although fixation was slightly different for both systems, motion artifacts were not observed which may be ascribed to the use of a single-shot MRI sequence. All animals survived the MRI examination. For further details of animal preparation see Boretius et al. (2004).

\subsection{DTI using single-shot STEAM}

Extending earlier versions with diffusion-encoding gradients applied to every individual stimulated echo (Merboldt et al., 1985, 1992), actual DW single-shot STEAM MRI sequences combine a spin-echo preparation period for diffusion encoding with a single-shot stimulated echo imaging module using RF readout pulses with low flip angles (Nolte et al., 2000; Boretius et al., 2004; Rieseberg et al., 2005). A schematic diagram is shown in Fig. 1. The flip angle $\alpha$ is calculated in accordance with the desired resolution in the phase-encoding direction which is determined by the corresponding point-spread function. Its full width at half-maximum $\mathrm{FWHM}_{\mathrm{ph}}$ is given by:

$\mathrm{FWHM}_{\mathrm{ph}}=\frac{2}{\pi} \mathrm{FOV}_{\mathrm{ph}}\left[\frac{T R_{\mathrm{i}}}{T_{1}}-\ln \cos \alpha\right]$ where $\mathrm{FOV}_{\mathrm{ph}}$ is the field-of-view in the phase-encoding direction and $\mathrm{TR}_{\mathrm{i}}$ is the repetition time of the imaging part of the single-shot STEAM sequence (Nolte et al., 2000). When replacing the full Fourier scheme by half Fourier phase-encoding, that is by using a lower number of phase-encoding steps and corresponding RF excitations/readout intervals, the flip angle $\alpha$ can be increased to yield a significant SNR improvement without sacrificing spatial resolution (Finsterbusch and Frahm, 2002; Boretius et al., 2004; Rieseberg et al., 2005). Similarly, the longer $T_{1}$ relaxation time at the higher field also allows for a higher flip angle without compromising the point-spread function. As far as the decreased $T_{2}$ relaxation time at higher field strength is concerned, single-shot STEAM MRI is only affected during the initial spin-echo diffusion preparation.

\section{3. $M R I$}

Half Fourier DW single-shot STEAM MRI was implemented on a $7 \mathrm{~T}$ animal MRI system (Bruker Pharmascan, Ettlingen, Germany) equipped with $300 \mathrm{mT} \mathrm{m}^{-1}$ gradients and a quadrature birdcage coil ( $20 \mathrm{~mm}$ i.d.) in transmit-receive mode. The sequence design followed previous developments at a lower field (Boretius et al., 2004) but employed a modified scheme for the diffusion gradients and different b values (see below). For comparison, similar measurements were performed at 2.35 T (Bruker DBX system, Ettlingen, Germany) using $100 \mathrm{mT} \mathrm{m}^{-1}$ gradients and a $100 \mathrm{~mm}$ Helmholtz coil for RF excitation in conjunction with an elliptical surface coil $(20 \mathrm{~mm} \times 12 \mathrm{~mm}$ i.d. $)$ for signal reception.

Diffusion preparation involved two $b$ values (10 and $1000 \mathrm{~s} \mathrm{~mm}^{-2}$ ) in 12 different directions (6 different gradients and their opposite values). At $7 \mathrm{~T}$, the combination of diffusionencoding gradients with a duration of $2.7 \mathrm{~ms}$ and a diffusion time $\Delta$ of $15 \mathrm{~ms}$ resulted in a spin-echo time TESE of $22.3 \mathrm{~ms}$ (compare Fig. 1). The imaging part of the STEAM sequence was characterized by a receiver bandwidth of $390 \mathrm{~Hz} /$ pixel, a stimulated echo time TE of $3.8 \mathrm{~ms}$, a repetition time $\mathrm{TR}_{\mathrm{i}}$ of $4.3 \mathrm{~ms}$ and a flip angle of the readout pulses of $12^{\circ}$. Altogether, the acquisition time of a single DW single-shot STEAM image was $309 \mathrm{~ms}$. While a Blackman-Harris pulse shape was employed for the low-flip angle readout RF pulses, all other RF pulses were sinc-shaped. A slice thickness of $720 \mu \mathrm{m}$ was complemented by a truly acquired in-plane resolution of $117 \mu \mathrm{m} \times 117 \mu \mathrm{m}$. This was accomplished by covering a $13.8 \mathrm{~mm} \times 15.0 \mathrm{~mm}$ rectangular FOV with a $118 \times 128$ matrix which was further reduced to a $64 \times 128$ matrix by half Fourier encoding with nine central lines.

Regional SNR values of the images were calculated from six different ROIs per measurement defined on images obtained with a low $b$ value. Mean values of the fractional anisotropy (FA) of the cortex and corpus callosum were obtained from respective maps and determined by averaging data of anatomically defined ROIs for each mouse and measurement. The main diffusion direction of the tissue water, which is commonly assumed to represent the direction of the underlying (fiber) structure, was computed as the largest eigenvector of the diffu- 


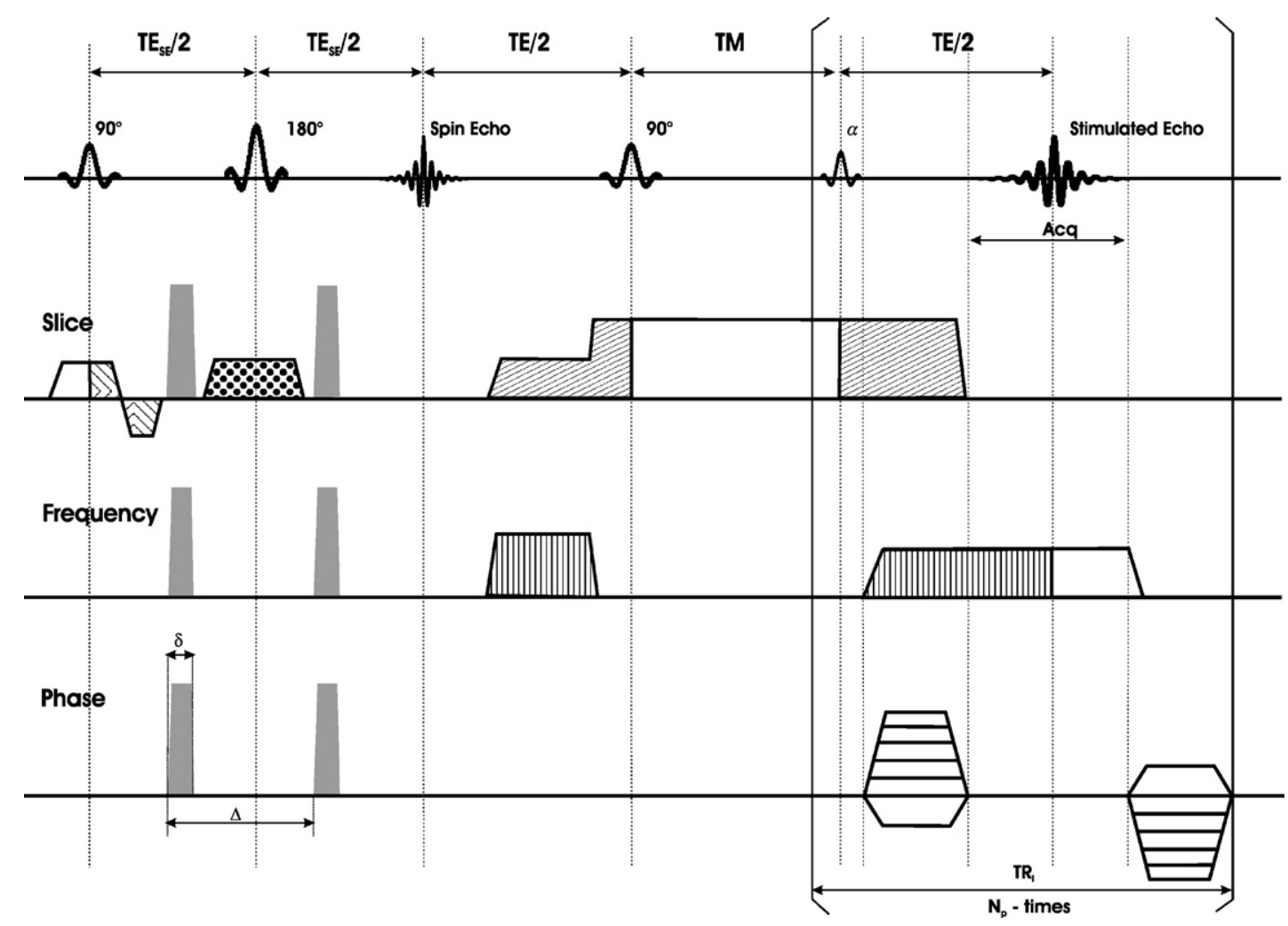

Fig. 1. Diffusion-weighted single-shot STEAM MRI combines a spin-echo preparation period for diffusion encoding (gradient duration $\delta$, diffusion time $\Delta$, spin-echo time $\mathrm{TE}_{\mathrm{SE}}$ ) with a single-shot stimulated echo imaging module (middle interval TM and stimulated echo time TE). The brackets indicate the repetitive readout interval for $\mathrm{N}_{\mathrm{p}}$ phase-encoding steps (RF pulses with low flip angle $\alpha$, repetition time $\mathrm{TR}_{\mathrm{i}}$ ). Refocusing gradient lobes for slice selection that belong together carry the same pattern. The refocusing phase-encoding gradients in the repetitive readout interval are utilized in conjunction with RF spoiling applied to the series of low-flip angle RF pulses.

sion tensor. Corresponding maps were color-coded according to anatomical orientations $($ red $=$ left-right, blue $=$ rostral-caudal and green $=$ dorsal-ventral) and superimposed onto the corresponding FA maps using the same post-processing strategies as detailed previously (Boretius et al., 2004).

\subsection{Fiber tracking}

Preprocessing of the diffusion data involved a linear interpolation by doubling the acquisition matrix to twice the resolution in all three dimensions, and a subsequent application of a threedimensional Gaussian filter ( $\sigma=1$ voxel). The calculation of the diffusion tensor employed a weighted linear least-squares algorithm. Estimates of neuroaxonal fiber projections were computed by the fiber assignment by continuous tracking (FACT) algorithm (Mori et al., 1999) after subdividing each start voxel to contain nine seed points. Criteria for terminating the tracking of individual fibers included an anisotropy threshold (i.e., FA values below 0.15 ) and a maximum stiffness condition. In the latter case, empirical optimization resulted in a termination of mouse fiber tracks when the diffusion directions in consecutive steps differed by more than $20^{\circ}$. The DTI analysis and visualization of fiber tracks relied on software developed in-house and followed the same principles as recently applied to a comprehensive analysis of the human corpus callosum (Hofer and Frahm, 2006).

\section{Results}

In comparison to previous studies at a field strength of $2.35 \mathrm{~T}$, the experimental parameters for the implementation of half Fourier DW single-shot STEAM MRI at $7 \mathrm{~T}$ required a number of adjustments to benefit from differences in hardware and relaxation times. For example, the higher gradient strength available at $7 \mathrm{~T}$ allowed for a shorter duration of the diffusion-encoding gradients. The concomitant reduction of the spin-echo time helped to alleviate the slightly stronger signal attenuation due to the shorter $T_{2}$ relaxation time. Because of the longer $T_{1}$ values at high fields, the repetition time had to be increased from 5 to $7.5 \mathrm{~s}$ to minimize saturation. The longer TR allows for the acquisition of up to 24 sections which are needed when fiber tractography requires to cover a large FOV with thin sections. Together, the short repetition time $\mathrm{TR}_{\mathrm{i}}$ due to the increased bandwidth at $7 \mathrm{~T}$, the higher flip angle of the readout pulses, the longer $T_{1}$ and the increased longitudinal magnetization resulted in a pronounced increase of the overall SNR. As shown in Fig. 2 this signal gain was successfully used to reduce the measurement time for a DTI data set at $150 \mu \mathrm{m} \times 150 \mu \mathrm{m} \times 1000 \mu \mathrm{m}$ resolution from about $3 \mathrm{~h}$ at $2.35 \mathrm{~T}$ ( 58 averages) to $23 \mathrm{~min}$ at $7 \mathrm{~T}$ ( 5 averages).

Noteworthy, for STEAM MRI no susceptibility artifacts were observed at either field strength. Typically, such artifacts refer to signal losses and/or geometric distortions at the base of the rodent brain near air-tissue interfaces. For a measurement time 


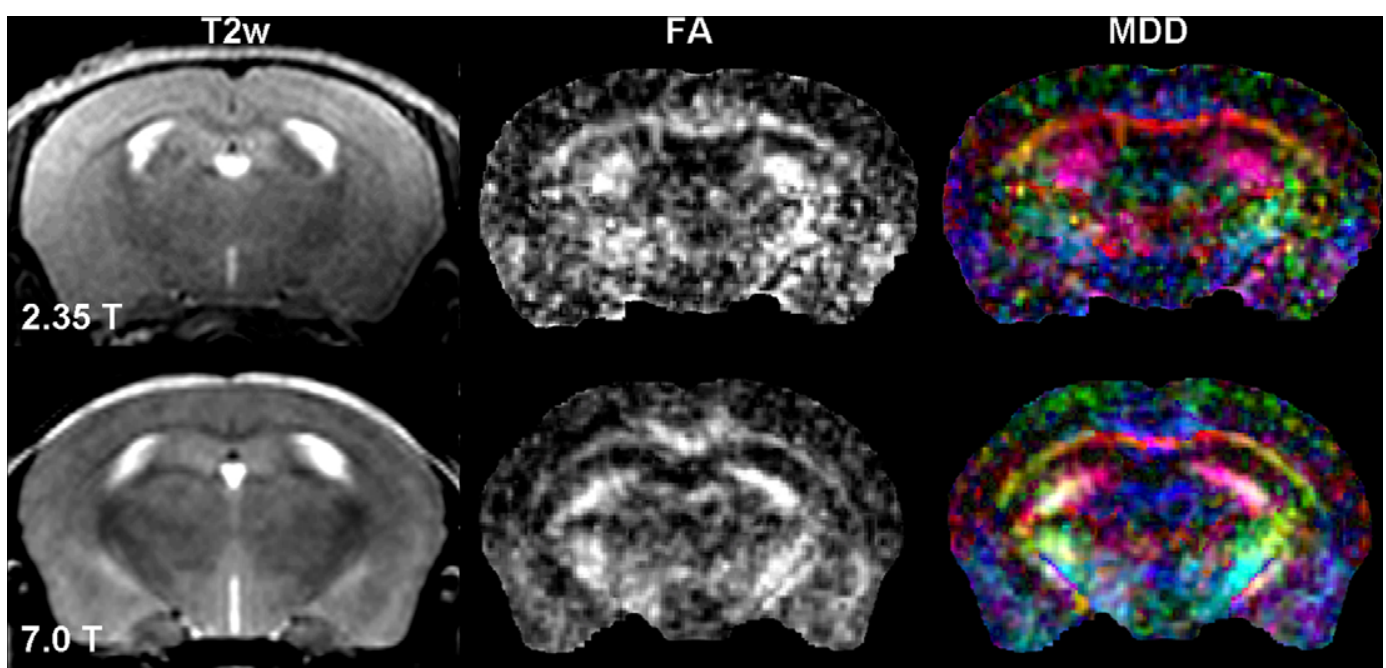

Fig. 2. $T_{2}$-weighted images $(\mathrm{T} 2 \mathrm{w})$ as well as corresponding maps of the fractional anisotropy (FA) and color-coded main diffusion direction (MDD) at $150 \mu \mathrm{m} \times 150 \mu \mathrm{m} \times 1000 \mu \mathrm{m}$ resolution obtained at $7 \mathrm{~T}$ (measuring time $23 \mathrm{~min}, 5$ averages) and $2.35 \mathrm{~T}$ (measuring time 179 min, 58 averages). The color codes for fiber orientation: red $=$ left-right, blue $=$ rostral-caudal and green $=$ dorsal-ventral .

Table 1

Experimental results for STEAM DTI of mouse brain in vivo at $7 \mathrm{~T}$

\begin{tabular}{lllll}
\hline Measuring time (min) & Resolution $\left(\mu \mathrm{m}^{3}\right)$ & SNR $^{\mathrm{a}}$ & FA $^{\mathrm{b} / \text { corpus callosum }}$ & FA/cortex \\
\hline 23 & $150 \times 150 \times 1000$ & $18 \pm 2$ & $0.45 \pm 0.06$ & $0.24 \pm 0.03$ \\
176 & $117 \times 117 \times 720$ & $23 \pm 2$ & $0.51 \pm 0.03$ & $0.22 \pm 0.02$ \\
\hline
\end{tabular}

a SNR: signal-to-noise ratio at $b=10 \mathrm{~s} \mathrm{~mm}^{-2}$.

${ }^{\mathrm{b}}$ FA: fractional anisotropy.

of about $3 \mathrm{~h}$ ( 38 averages), the spatial resolution of the DTI acquisition at $7 \mathrm{~T}$ could be increased to $117 \mu \mathrm{m} \times 117 \mu \mathrm{m} \times 720 \mu \mathrm{m}$ voxel size which resulted in a substantial improvement of FA and MDD maps as demonstrated in Fig. 3. Noteworthy, the upper two sections are in the vicinity of the ear cavities and affected by susceptibility differences but do not cause any visible artifacts in the STEAM images. Quantitative results for acquisitions at the two different resolutions are summarized in Table 1. While cortical FA values are very similar, the better resolution reveals a tendency for slightly higher FA values in the corpus callosum. This finding may be explained by reduced partial volume averaging with neighboring, less anisotropic tissue.

Finally, the high-resolution DTI maps were further exploited for tracking of major fiber projections in the mouse brain in vivo. Fig. 4 shows 3D reconstructions of the axonal connectivity patterns involving the corpus callosum and anterior commissure in multiple view orientations. Again, the color codes for the local fiber orientation.

\section{Discussion}

The advantages predicted for DW single-shot STEAM MRI at higher magnetic fields were experimentally realized with substantial practical gains. For a threefold increase in field strength as used here, either the measuring time could be reduced by a factor of 8 or the spatial resolution could be increased by a factor of 2.3. Most importantly, the achieved quality of the DTI maps and the respective directional information turned out to be sufficient for fiber tractography of the main axonal projections in the mouse brain. While DTI-derived connectivity information has already been obtained for mouse brain ex vivo (Mori et al., 2001; Zhang et al., 2002) and in a single case also in vivo (Lin et al., 2005), this study presents the first in vivo fiber tracts from the corpus callosum and anterior commissure of a living mouse. Further technical improvements in terms of SNR and/or spatial resolution are to be expected by using adapted surface or array coils rather than a transmit/receive birdcage coil or even higher magnetic field strengths.

Most importantly, for STEAM MRI sequences the established gain in performance may be obtained without any compromise due to geometric distortions or signal losses. In high-field applications, this practical advantage over EPI may even compensate for the loss of half of the magnetization in a STEAM sequence. Previous (comparative) studies indicated the pronounced shortening of $T_{2}^{*}$ at higher fields to cause severe geometric distortions in acquisitions based on EPI (Farzaneh et al., 1990). To overcome these limitations, it has been proposed to reduce the EPI echo train length by segmented multishot techniques (Butts et al., 1997) or partially parallel imaging (Jaermann et al., 2006). Although pertinent strategies certainly bear some advantages, parallel acquisitions are at the expense of SNR and eventually may also degrade image quality, while multishot EPI looses the basic snapshot character of a highspeed MRI technique and therefore becomes prone to motion 

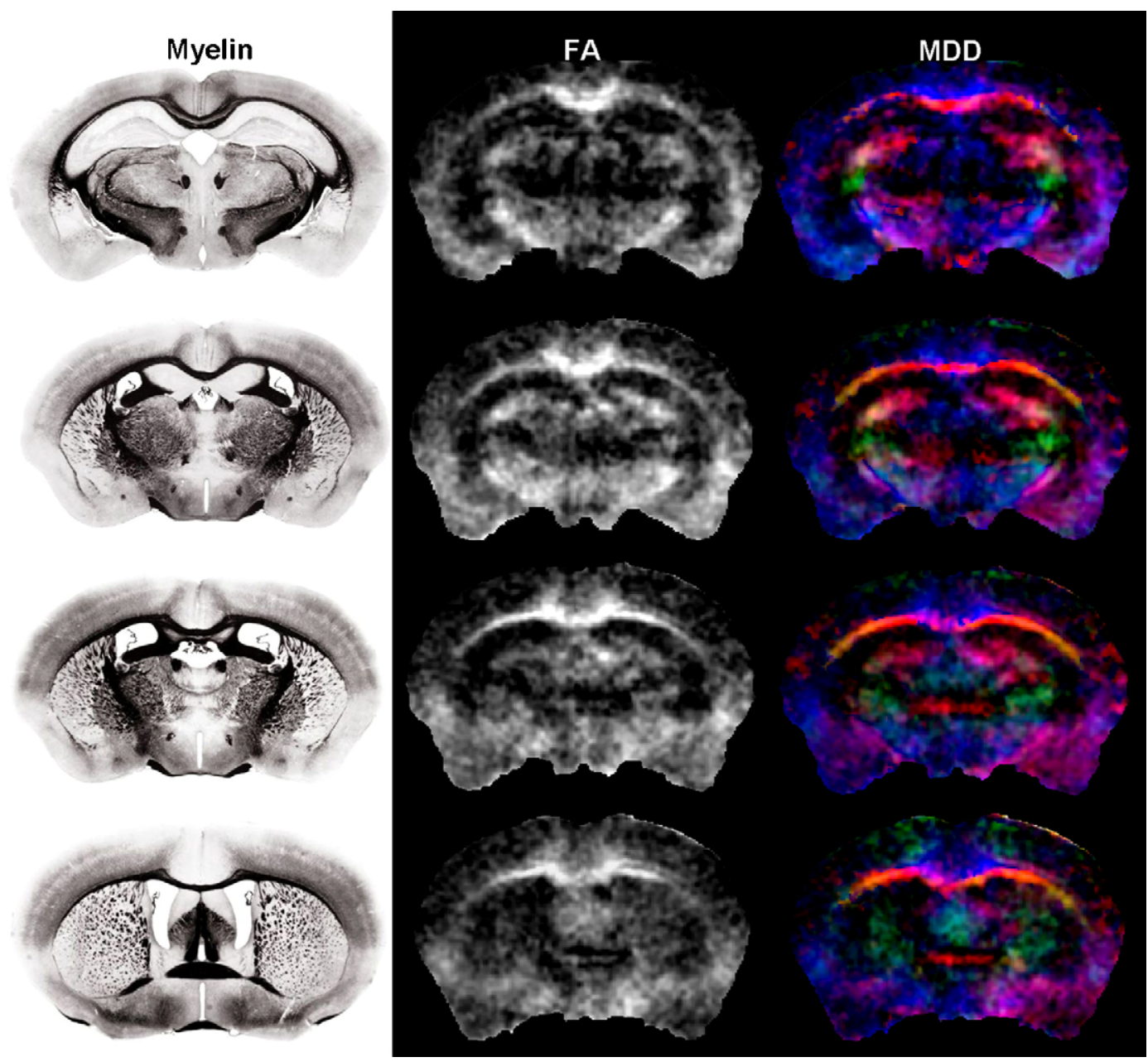

Fig. 3. Histological sections (posterior-anterior) stained for myelin (courtesy: www.hms.harvard.edu) as well as corresponding $7 \mathrm{~T}$ maps of the fractional anisotropy (FA) and color-coded main diffusion direction (MDD) at $117 \mu \mathrm{m} \times 117 \mu \mathrm{m} \times 720 \mu \mathrm{m}$ resolution (measuring time 176 min, 38 averages). Same color code as in Fig. 2.

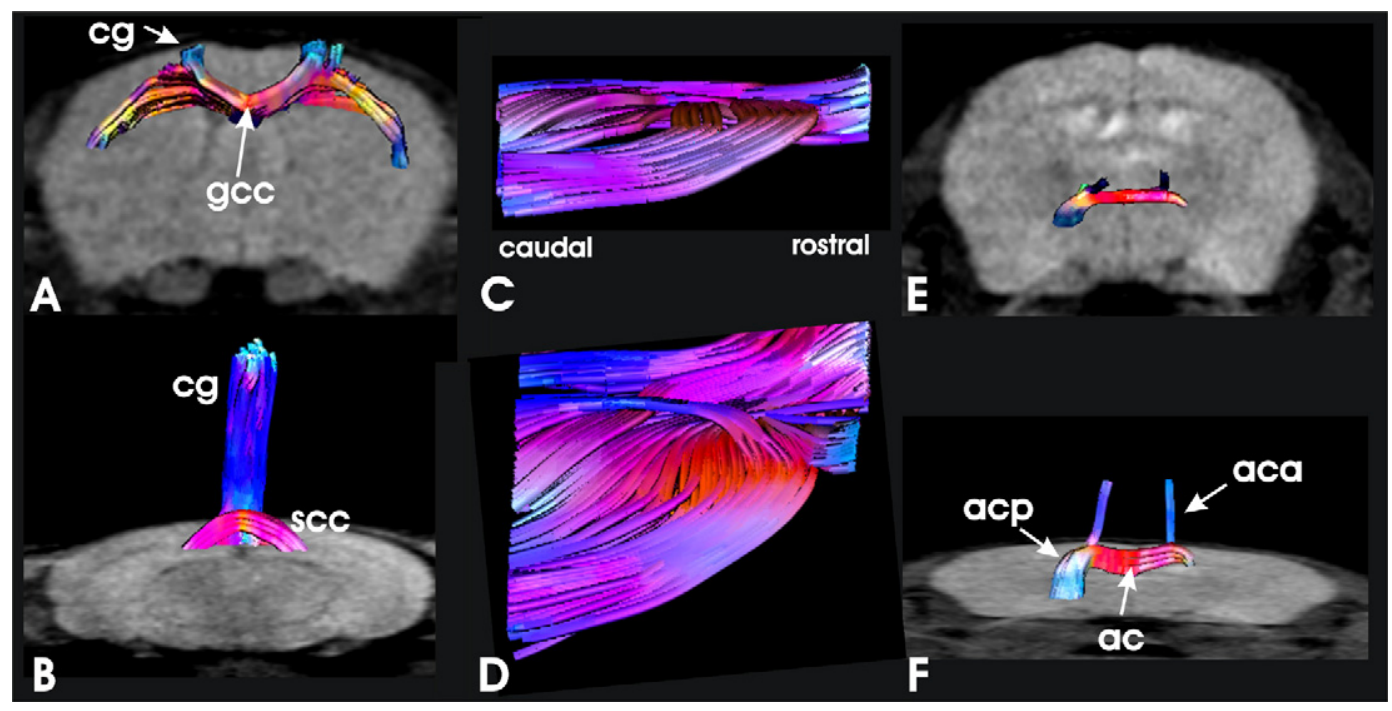

Fig. 4. Fiber tracts from a mouse brain in vivo at $7 \mathrm{~T}$ overlaid onto the mildly diffusion-weighted images $\left(b=10 \mathrm{~s} \mathrm{~mm}^{-2}\right)$. (A) Oblique ventral view of a $3 \mathrm{D}$ reconstruction of the frontal part of the corpus callosum (cg: cingulum and gcc: genu of corpus callosum), (B) ventral view of cingulum and splenium of corpus callosum (scc), (C) right-left, (D) oblique right-left view of the corpus callosum, (E) frontal and (F) ventral view of the anterior commissure (ac, acp: posterior part and aca: anterior part). Same color code as in Fig. 2. 
artifacts (Jiang et al., 2002). Alternatively, RF-refocused spinecho signals may be used in a DW fast spin-echo MRI sequence. In human applications, this approach commonly suffers from residual phase errors arising from unavoidable movements that need to be corrected for by navigator techniques (Mori and van Zijl, 1998). This effect may be less prominent when studying anesthetized and artificially ventilated animals with fixation. In either case, however, the use of a relatively large number of $180^{\circ}$ pulses may exceed the limits for the specific absorption rate, so that the use of DW fast-spin echo MRI may become a safety concern at high fields.

In summary, DW single-shot STEAM MRI seems to be a promising tool for high-field DTI of mouse brain in vivo. The achieved resolution and image quality offers interesting future perspectives for a variety of applications addressing the microstructural properties of the brain tissue including the identification of specific white matter fiber tracts. Particular emphasis is paid on the evaluation of neuroaxonal fiber architecture in animal models of neurodegeneration and neuroinflammation.

\section{Acknowledgments}

This study is supported by the Institut für Multiple-SkleroseForschung, Bereich Humanmedizin der Universität Göttingen und Gemeinnützige Hertie-Stiftung. The authors thank Matthias Küntzel for providing his fiber tracking software.

\section{References}

Basser PJ, Mattiello J, LeBihan D. Estimation of the effective self-diffusion tensor from the NMR spin echo. J Magn Reson B 1994;103:247-54.

Boretius S, Natt O, Watanabe T, Tammer R, Ehrenreich L, Frahm J, et al. In vivo diffusion tensor mapping of the brain of squirrel monkey, rat, and mouse using single-shot STEAM MRI. MAGMA 2004;17:339-47.

Butts K, Pauly J, DeCrespigny A, Moseley M. Isotropic diffusion-weighted and spiral-navigated interleaved EPI for routine imaging of acute stroke. Magn Reson Med 1997;38:741-9.

Farzaneh F, Riederer SJ, Pelc NJ. Analysis of T2 limitations and off-resonance effects on spatial resolution and artifacts in echo-planar imaging. Magn Reson Med 1990;14:123-39.

Frahm J, Haase A, Matthaei D, Merboldt KD, Hänicke W. Rapid NMR imaging using stimulated echoes. J Magn Reson 1985;65:130-5.

Finsterbusch J, Frahm J. Half-Fourier single-shot STEAM MRI. Magn Reson Med 2002;47:611-5.
Hofer S, Frahm J. Topography of the human corpus callosum revisitedcomprehensive fiber tractography using magnetic resonance diffusion tensor imaging. Neuroimage 2006;32:989-94.

Huisman TA, Schwamm LH, Schaefer PW, Koroshetz WJ, Shetty-Alva N, Ozsunar Y, et al. Diffusion tensor imaging as potential biomarker of white matter injury in diffuse axonal injury. Am J Neuroradiol 2004;25:370-6.

Jaermann T, Pruessmann KP, Valavanis A, Kollias S, Boesiger P. Influence of SENSE on image properties in high-resolution single-shot echo-planar DTI. Magn Reson Med 2006;55:335-42.

Jiang H, Golay X, van Zijl PCM, Mori S. Origin and minimization of residual motion-related artifacts in navigator-corrected segmented diffusionweighted EPI of the human brain. Magn Reson Med 2002;47:818-22.

Lin CY, Sun SW, Hong CY, Chang C. Unsupervised identification of white matter tracts in a mouse brain using a directional correlation-based region growing (DCRG) algorithm. Neuroimage 2005;28:380-8.

Merboldt KD, Hänicke W, Frahm J. Self-diffusion NMR imaging using stimulated echoes. J Magn Reson 1985;64:479-86.

Merboldt KD, Hänicke W, Gyngell ML, Bruhn H, Frahm J. Diffusion imaging of the human brain in vivo using high-speed STEAM MRI. Magn Reson Med 1992;23:179-92.

Mori S, van Zijl PCM. A motion correction scheme by twin-echo navigation for diffusion-weighted magnetic resonance imaging with multiple rf echo acquisition. Magn Reson Med 1998;40:511-6.

Mori S, Crain BJ, Chacko VP, van Zijl PC. Three-dimensional tracking of axonal projections in the brain by magnetic resonance imaging. Ann Neurol 1999;45:265-9.

Mori S, Itoh R, Zhang J, Kaufmann WE, van Zijl PCM, Solaiyappan M, et al. Diffusion tensor imaging of the developing mouse brain. Magn Reson Med 2001;46:18-23.

Nair G, Tanahashi Y, Low HP, Billings-Gagliardi S, Schwartz WJ, Duong TQ. Myelination and long diffusion times alter diffusion-tensor-imaging contrast in myelin-deficient shiverer mice. Neuroimage 2005;28:165-74.

Nolte UG, Finsterbusch J, Frahm J. Rapid isotropic diffusion mapping without susceptibility artefacts: whole brain studies using diffusion-weighted singleshot STEAM MR imaging. Magn Reson Med 2000;44:731-6.

Rieseberg S, Merboldt KD, Küntzel M, Frahm J. Diffusion tensor imaging using partial Fourier STEAM MRI with projection onto convex subsets reconstruction. Magn Reson Med 2005;54:486-90.

Rutt BK, Lee DH. The impact of field strength on image quality in MRI. Magn Reson Imaging 1996;6:57-62.

Song SK, Sun SW, Ramsbottom MJ, Chang C, Russell J, Cross AH. Dysmyelination revealed through MRI as increased radial (but unchanged axial) diffusion of water. Neuroimage 2002;17:1429-36.

Sun SW, Liang HF, Trinkaus K, Cross AH, Armstrong RC, Song SK. Noninvasive detection of cuprizone induced axonal damage and demyelination in the mouse corpus callosum. Magn Reson Med 2006;55:302-8.

Zhang J, van Zijl PCM, Mori S. Three-dimensional diffusion tensor magnetic resonance microimaging of adult mouse brain and hippocampus. Neuroimage 2002;15:892-901. 